\title{
FRECUENCIA DE HEMATOZOARIOS EN BOVINOS DE UNA REGIÓN DEL CARIBE COLOMBIANO
}

\section{BOVINE HEMOPARASITES FREQUENCY FROM COLOMBIAN CARIBBEAN REGION}

\author{
Alfonso Calderón ${ }^{1}$ Nicolás Martinez ${ }^{2}$, Haydée Iguarán ${ }^{3}$
}

\begin{abstract}
${ }^{1}$ MVZ, M.Sc., Docente Instituto de Investigaciones Biológicas del Trópico, Facultad de Medicina Veterinaria y Zootecnia. Universidad de Córdoba, kilómetro 27 vía Ciénaga de Oro, Córdoba, Colombia. Estudiante Doctorado en Medicina Tropical Sue-Caribe. Universidad de Cartagena, e-mail: acalderonr@correo.unicordoba.edu.co; ${ }^{2}$ MVZ, M.Sc., Docente Facultad de Medicina Veterinaria y Zootecnia. Universidad de Córdoba, kilómetro 27 vía Ciénaga de Oro, e-mail: namatrinez@correo. unicordoba.edu.co; ${ }^{3}$ MVZ, Especialista en Laboratorio Clínico, Profesional Especializado, Facultad de Medicina Veterinaria y Zootecnia. Universidad de Córdoba, kilómetro 27 vía Ciénaga de Oro, e-mail: higuarandiaz@correo.unicordoba.edu.co
\end{abstract}

Rev. U.D.C.A Act. \& Div. Cient. 19(1): 131-138, Enero-Junio, 2016

\section{RESUMEN}

Los hematozoarios han limitado la producción ganadera en regiones tropicales, cuyas características climáticas brindan nichos ecológicos, propicios para el desarrollo de artrópodos, que son vectores de varias especies de los géneros, como: Babesia, Anaplasma y Trypanosoma. Los hematozoarios producen pérdidas económicas directas e indirectas con implicaciones en el comercio. El objetivo fue determinar la frecuencia de hematozoarios en bovinos de una región del Caribe colombiano. Mediante un estudio descriptivo de corte transversal y con un muestreo por conveniencia, se seleccionaron doce fincas, en las que se tomaron muestras de sangre de 310 bovinos de diferentes edades, sexo y grupo racial, para el diagnóstico de hematozoarios por observación directa y determinación de algunos valores hemáticos. La mayor frecuencia de hematozoarios fue para anaplasmosis, con el $27,74 \%$, seguido de babesiosis, con el $19,35 \%$ y tripanosomiasis, con el $2,26 \%$, lo que indica que esta zona sigue siendo endémica, para los hematozoarios.

Palabras clave: Anemia, Anaplasma, Babesia, parasitemía, Trypanosoma.

\section{SUMMARY}

The haematozoa have limited animal production in tropical regions, the climatic characteristics in tropical regions provide ecological niches for the development of arthropods which are vectors of several species of the genres as Babesia, Anaplasma and Trypanosoma. The haematozoa produce direct and indirect economic losses with implications on trade. The objective was to determine the frequency of haematozoa in cattle in the colombian Caribbean region. A descriptive cross-sectional study with a convenience sample, 12 farms were selected, where 310 blood samples from cattle of different ages, sex, racial group were taken, for the diagnosis of haematozoa by direct observation of extended blood and some hematology values. The highest frequency of haematozoa was for anaplasma sp. with $27.74 \%$, followed by babesia sp. with $19.35 \%$ and trypanosoma sp. of $2.26 \%$. It is indicating that this area is still endemic for haematozoa.

Key words: Anemia, Anaplasma, Babesia, parasitemia, Trypanosoma.

\section{INTRODUCCIÓN}

Babesia bigemina, B. bovis, B. divergens, B. major, $B$. ovata han sido reportadas en bovinos, como causantes de la babesiois bovina (Bock et al. 2004). Son protozoarios de ubicación intraheritrocitarios, del Sub-Phylum Apicomplexa, Clase Piroplasmasida, Orden Hemosporidia, Sub-Orden Piroplasmidea, Familia Babesidae (Quijada et al. 1998), que se caracteriza por producir anemia hemolítica, fiebre, hemoglobinuria, disminución de la producción y, en casos extremos, la muerte (Ristic \& McIntyre, 1981). Su transmisión está determinada por la picadura de la garrapata Rhipicephalus microplus, ectoparásito que habita zonas tropicales y subtropicales, que poseen las condiciones climáticas y ecológicas, propicias para el mantenimientos de estos artrópodos (Regassa et al. 2003). Pueden afectar a muchas especies de mamíferos, con un impacto importante en el ganado bovino (Bock et al. 2004; Zintl et al. 2003).

La anaplasmosis bovina es producida por Anaplasma marginale (Añez et al. 2010), género de la familia Analasmata- 
ceae del orden Rickettsiales; se trasmite por vectores artrópodos, como moscas y garrapatas o por vía iatrogénica y en bovinos, con infección persistente, actúan como reservorios para la trasmisión por garrapatas. Esta enfermedad se caracteriza por anemia progresiva e ictericia, pérdida de peso, debilidad, inapetencia, fiebre, cesa de la rumia, no hay hemoglobinemia ni hemoglobinuria; el ganado infectado puede permanecer como portador (OIE, 2012; Howarth et al. 1972; Wickwite et al. 1987).

Trypanosoma vivax, protozoario hemático que produce la tripanosomosis bovina, cuya trasmisión se produce por insectos hematófagos del género Tabanus (Diptera: Tabanidae) o iatrogénicamente y signos, que incluyen: fiebre, anemia severa, pérdidas significativas en la producción y la condición corporal y trastornos reproductivos. La infección puede progresar de carácter agudo a crónico; en muchos casos, produce la muerte por las alteraciones producidas en los órganos y la química sanguínea (Rossi et al. 2008; Suarez et al. 2009).

Estas enfermedades hematozoarias han limitado la producción ganadera en diferentes regiones tropicales y se han constituido en un riesgo potencial en ganaderías especializadas en la producción de leche, ya que ha reportado el hallazgo de $R$. microplus, a 2.903 metros sobre el nivel del mar (msnm), vector para la babesiosis y anaplasmosis bovina (Vizcaíno, 2011; Bolívar, 2013).

Las regiones tropicales, por sus características climáticas de humedad, de temperatura y de luminosidad, brindan nichos ecológicos, propicios para el desarrollo de garrapatas, de moscas picadoras y de tábanos, que son vectores de hematozoarios, como Babesia, Anaplasma y Trypanosoma (Prada \& Crespo, 2006; Benavides et al. 2012).

El calentamiento global ha generado alteración de los regímenes de temperatura y los patrones de lluvia, lo que ha permitido cambios en la distribución y en la frecuencia de los ectoparásitos vectores de hematozoarios, en diversas latitudes de áreas tropicales y subtropicales, lo que, a su vez, resulta en un incremento de la probabilidad de transmisión de enfermedades; sin embargo, hay otras variables que pueden explicar estos cambios, como la explosión y los movimientos demográficos, las condiciones socioeconómicas, las alteraciones del paisaje y el comercio global. Igualmente, las actividades antrópicas podrían tener una influencia más determinante y profunda sobre la distribución de garrapatas y de moscas, en comparación con el cambio climático (Cortés, 2010; Thornton et al. 2009).

Dentro de las medidas de prevención y de control, se destaca la aplicación de sustancias de origen químico contra los vectores, pero la presencia de estos rastros o residuos lleva a implicaciones en el comercio, ya que afectan la inocuidad de alimentos de origen pecuario (Márquez, 2008).

Estas enfermedades hematozoarias influyen en los programas de mejoramiento bovino en el trópico, por las restricciones en el uso de razas Bos taurus, con el objetivo de aumentar la productividad de las razas $B$. indicus, siendo las $B$. taurus más susceptibles a infestaciones por ectoparásitos, protozoarios y rickettsias (González \& Meléndez, 2007); aunque en África, se han hallado bovinos de razas B. taurus tolerantes al tripanosoma (Gachohi et al. 2009). En Venezuela, bovinos de la raza criollo limonero puros y mestizo fueron tolerantes al tripanosoma, lo que permite una mayor adaptación a las condiciones tropicales (Agudo et al. 2009).

En Colombia, la garrapata, vector de babesias y anaplasmas, se localiza en altitudes inferiores a los $2.200 \mathrm{msnm}$ y temperaturas que fluctúa entre los 28 y $32^{\circ} \mathrm{C}$, con una humedad relativa entre 85 y 90\% (Ríos et al. 2010); sin embargo, ya existen reportes donde describen la presencia de babesiosis bovina en zonas ubicadas a mayores altitudes (Betancourt, 2011) y han relacionado esto con el efecto del cambio climático, que favorece en la adaptación de las garrapatas.

Alteraciones ambientales en los últimos años han ocasionado cambios en los regímenes de lluvias, incremento de la temperatura ambiental y de la humedad relativa; esto ha provocado en el Caribe colombiano disminución o pérdida de la oferta forrajera, detrimento del nivel freático de los suelos, ampliación de la época seca, lo que incide directamente en un desbalance nutricional y del bienestar animal, generando reducción en la condición corporal, bajas en la producción de carne y de leche, desequilibrio de la estabilidad enzoótica y baja en el sistema inmune de los bovinos, que se traduce en un aumento de la susceptibilidad al ataque de los ectoparásitos, vectores de hematozoarios. Actualmente, en Córdoba, en Sucre y en la región del Urabá, no se registran reportes de afectación por hematozoarios en bovinos; por lo tanto, el objetivo del presente estudio fue determinar la frecuencia de hematozoarios en bovinos, en una época seca, en una región del Caribe colombiano.

\section{MATERIALES Y MÉTODOS}

Tipo de estudio. Se implementó un estudio descriptivo, de corte transversal (Manterola \& Otzen, 2014).

Selección de la muestra. Por medio de un muestreo por conveniencia, se seleccionaron en 8 municipios de tres departamentos (Tabla 1), 12 empresas ganaderas, donde en cada una de ellas, se tomó entre 19 a 25 bovinos, a los cuales, se les colectó una muestra de sangre. Los criterios de inclusión fueron: que se permitirá por propietarios y administradores el proceso de toma de muestras, de registros productivos 
y reproductivos. Igualmente, se implementó una encuesta para conocer aspectos de manejo y de conocimientos sobre hematozoarios; este muestreo, se realizó durante una época de mínima precipitación.

Toma de las muestras. En cada una de las empresas ganaderas, se tomaron tres milímetros de sangre en tubos vacutainer al vacío sin anticoagulante y con anticoagulante (EDTA), de la vena coccígea, previa desinfección de esta área; cada muestra se rotuló y se conservó en refrigeración $4^{\circ} \mathrm{C}$, en cavas de poliestireno y remitidas hasta la Clínica Ju- lio E. Cuervo de la Universidad de Córdoba, sede Berástegui, donde se procesaron. Además de la identificación de los bovinos, se tomaron algunos anamnesicos clínicos y la evaluación de la condición corporal (CC); se calificó entre 1 y 5 y se categorizó como: condición excelente, $>4,25$; condición buena, 4,0; condición regular, cuando osciló entre 3,75 y 3,0 y condición mala, entre $<2,75$. La clasificación de los fenotipos se ajustó a la raza cebú (Brahman comercial) y mestizos los cruzamientos de cebú por Bos taurrus (Holstein, Simmental, Pardo Suizo).

Tabla 1. Ubicación de las empresas ganaderas, número de muestras de sangre para diagnóstico y frecuencia de los hematozoarios en bovinos, en el Caribe colombiano.

\begin{tabular}{|c|c|c|c|c|c|c|}
\hline \multirow{2}{*}{ Depto. } & \multirow{2}{*}{ Municipio } & \multirow{2}{*}{$\begin{array}{c}\text { No. } \\
\text { Fincas }\end{array}$} & \multirow{2}{*}{$\begin{array}{c}\text { No. de } \\
\text { muestras }\end{array}$} & \multicolumn{3}{|c|}{ Bovinos con hematozoarios } \\
\hline & & & & A. marginale & B. bigemina & T. vivax \\
\hline Antioquía & Arboletes & 1 & 25 & 5 & 4 & 0 \\
\hline \multirow{6}{*}{ Córdoba } & Ciénaga de Oro & 1 & 22 & 10 & 3 & 0 \\
\hline & Los Córdobas & 2 & 46 & 14 & 14 & 2 \\
\hline & Montería & 4 & 120 & 39 & 19 & 1 \\
\hline & P. Libertador & 1 & 19 & 4 & 3 & 0 \\
\hline & Planeta Rica & 1 & 22 & 5 & 7 & 0 \\
\hline & Valencia & 1 & 37 & 2 & 0 & 0 \\
\hline Sucre & San Benito Abad & 1 & 19 & 7 & 10 & 4 \\
\hline TOTAL & & 12 & 310 & 86 & 60 & 7 \\
\hline
\end{tabular}

Análisis de las muestras. Para hematocrito, se homogenizó la sangre del tubo con EDTA y por medio de un capilar, se llenó hasta las tres cuartas partes de la capacidad del tubo capilar y se selló el otro extremo con plastilina; se centrifugó el capilar a 13.680 gravedades, durante 5 min y se procedió a su lectura en la tabla para hematocrito. Los diagnósticos de hematozoarios, se efectuaron por medio de extendidos, donde una pequeña gota de sangre, tomada con una pipeta Pasteur, se depositó en una lámina portaobjetos y se expandió con un cubreobjetos, con una inclinación de $45^{\circ}$; el extendido se secó y después se aplicó una solución del colorante de Wright por toda la lámina, durante 2 min; luego, se colocaron 2 o 3 gotas de agua destilada, se sopló hasta que la placa cambió el color tornasol, se dejó durante 2 min, se enjuagó con agua corriente [fluctúa entre los 28 y $32^{\circ} \mathrm{C}$, con una humedad relativa entre 85 y 90\% (Latimer et al. 2005)] y se procedió a la lectura, donde se leyeron 10.000 eritrocitos y el resultado se expresó en porcentaje, para Babesia y Anaplasma; para tripanosomas, se evaluaron 100 campos y el resultado se expresó por campo; todas las lecturas se hicieron en un microscopio de luz con el objetivo de 100X. Se consideraron animales con anaplasma, cuando el por- centaje de parasitemía fue superior al 0,03\% y la presencia de babesia y tripanosoma como bovinos, como infectado o con hematozoarios.

Análisis estadístico. Se elaboró una base de datos en formato Excel, donde se consignó información de las variables evaluadas de los bovinos y se compararon entre bovinos sin hematozoarios y bovinos con hematozoarios; donde esta última categoría se estableció con, al menos, el diagnóstico de un solo grupo. La comparación de los grupos se implementó con la prueba de t-student para las variables cuantitativas; todos estos cálculos se efectuaron mediante el software SAS.

Aspectos éticos. El Comité de Ética del Instituto de Investigaciones Biológicas del Trópico (IIBT) de la Universidad de Córdoba, clasificó este estudio de bajo riesgo. Las muestras fueron tomadas por un Médico Veterinario Zootecnista, quien efectuó los procedimientos de la toma de las muestras, teniendo en cuenta los procedimientos de manejo y de conservación, las normas éticas, técnicas, científicas y administrativas para la investigación en animales, según la Ley 84 del Congreso de Colombia de 1989. A lo largo del 
estudio, se mantuvo la confidencialidad de la información; solo se proporcionó a los encargados del manejo directo de los bovinos.

\section{RESULTADOS Y DISCUSIÓN}

Al considerar como bovinos con hematozoarios, al menos cualquier individuo donde se observó algún agente etiológico evaluado, la frecuencia fue del 40,32\% y la principal asociación de patógenos fue Anaplasma y Babesia, con el 8,39\%. No hubo asociación de Babesia y de Trypanosoma y no se determinó ningún caso, donde los tres agentes etiológicos estuvieron presentes. En todas las empresas ganaderas y en todos los municipios, se comprobó la presencia de hematozoarios.

Mayores casos de detección se han obteniendo empleando técnicas de biología molecular, como lo reportado por Canever et al. (2014). Actualmente, se están empleando técnicas de biología molecular, donde la frecuencia o seroprevalencia son más elevadas, por la alta sensibilidad que la empleada en el actual estudio.

Además de cambio climático hay otros factores que pueden modificar distribuciones de garrapatas (Giles et al. 2014). Dentro de las variables evaluadas para el control de ectoparásitos, se encontró que en el 83,33\% de las fincas desconocen el ciclo biológico de las garrapatas, la dilución y la cantidad de solución aplicada por animal y no hacen rotación de moléculas o principios activos, a pesar de usar diferentes nombres comerciales. El 100\% de los productores o encargados directos del manejo desconocen la calidad fisicoquímica del agua y la correcta calibración de equipos de aspersión y no realizan muestreos para determinar el tipo de garrapatas o hematozoarios.
Se evaluaron 151 bovinos cebú y 159 mestizos (Tabla 2); se encontró que la mayor frecuencia de hematozoarios fue en el grupo de mestizos (23,55\%), en comparación con bovinos cebú (16,77\%), aunque estas diferencias no fueron significativas; igualmente, la misma tendencia se determinó para la variable categoría animal. Se conoce que ganados $B$. indicus son más resistentes a los ectoparásitos que los $B$. taurus (Bianchin et al. 2007) y con el cruzamiento de estas dos grupos, se produce un mestizaje, que permite el incremento de los índices productivos, debido al vigor hibrido o heterosis, generado en el cruce y mayor grado de resistencia a las condiciones tropicales, frente al $B$. taurus (Frisch et al. 2000; Silva et al. 2007; Oliveira et al. 2009).

Se determinó que la mayor frecuencia de hematozoarios fue para Anaplasma sp., con el 27,74\%, seguido de Babesia, 19,35\% y Trypanosoma sp., con el 2,26\% (Tabla 1). En la región del bajo Cauca y Alto San Jorge, se determinó que Anaplasma sp. fue el hematozoario más frecuente, con el 61,8\% (Herrera et al. 2008). En una región del Noreste de Brasil, se determinó que el mayor agente de hematozoarios fue $A$. marginale, con el $89,1 \%$, seguido de $B$. bovis, con el $68.8 \%$ y B. bigemina, con el 52.5\% (Souza et al. 2013).

Esta mayor frecuencia de Anaplasma sp., en el actual estudio, se puede deber a que el muestreo se efectuó en la época de mínima precipitación, donde hay mayor infestación de garrapatas y las condiciones ambientales disminuyen la disponibilidad de forrajes, situaciones que conducen a estrés, produciendo un aumento de infecciones por hematozoarios. Otras causas, como picaduras de moscas y factores iatrogénicos, como el no uso de agujas estériles por animal y el no cambio de guantes en chequeos reproductivos, han sido reportadas como causas de hematozoarios (Souza et al. 2013), variables que no fueron evaluadas en el presente estudio. En Costa Rica, la exposición del ganado por A. margin-

Tabla 2. Bovinos muestreados y frecuencia de bovinos con y sin presencia de hematozoarios, por tipo racial, en el Caribe colombiano.

\begin{tabular}{|c|c|c|c|c|c|c|c|c|c|c|}
\hline \multirow{2}{*}{ Categoría } & \multicolumn{4}{|c|}{ Bovinos muestreados } & \multicolumn{3}{c|}{ Bovinos con hematozoarios } & \multicolumn{2}{c|}{ Frecuencia } \\
\cline { 2 - 12 } & \multicolumn{2}{|c|}{ Cebún } & \multicolumn{2}{c|}{ Mestizos } & \multicolumn{2}{c|}{ Cebú } & \multicolumn{2}{c|}{ Mestizos } & \multicolumn{2}{c|}{} \\
\cline { 2 - 12 } & $\mathrm{n}$ & $\%$ & $\mathrm{n}$ & $\%$ & $\mathrm{n}$ & $\%$ & $\mathrm{n}$ & $\%$ & $\mathrm{n}$ & $\%$ \\
\hline Toros & 11 & 3,54 & 7 & 2,27 & 1 & 0,32 & 3 & 0,97 & 4 & 1,29 \\
\hline Toretes & 16 & 5,16 & 30 & 9,68 & 3 & 0,97 & 8 & 2,58 & 11 & 3,55 \\
\hline Vacas & 62 & 20,0 & 61 & 19,67 & 30 & 9,68 & 35 & 11,29 & 65 & 20,97 \\
\hline Terneros & 62 & 20,0 & 61 & 19,67 & 18 & 5,81 & 27 & 8,71 & 45 & 14,51 \\
\hline Total & 151 & 48,70 & 159 & 51,29 & 52 & 16,77 & 71 & 23,55 & 125 & 40,32 \\
\hline
\end{tabular}


ale es común en los hatos lecheros, donde la inestabilidad endémica es, probablemente, debida al inadecuado control de vectores (Oliveira et al. 2011).

En el actual estudio, la principal asociación fue anaplasma y babesia, con el 8,39\% y en Santa Catarina (Brasil), determinaron que el $54,5 \%$ de los animales estaban coinfectados con dos o tres parásitos, usando PCR multiplex (Canever et al. 2014). Una mayor tasa de seroprevalencia de $T$. evansi se asoció con una mayor prevalencia de tábanos (Cadioli et al. 2012).

En cuatro fincas, los propietarios o encargados del manejo directo de los bovinos manifestaron la muerte en días, previos a la toma de muestras y, en estas empresas, se determinó la presencia de Trypanosma sp. Una de las mayores causas de anemia en el ganado en zonas endémicas es la tripanosomiasis (Biryomumaisho et al. 2013).

Al comparar los promedios entre bovinos sin y con hematozoarios (Tabla 3), se presentaron diferencias significativas $(p<0.05)$, para los valores del hematocrito, hemoglobina, eritrocitos y leucocitos; estas diferencias, se pueden deber a lo reportado en Brasil, donde la anemia fue el cambio más importante que se evidenció en bovinos infectados y mostraron promedios significativos más bajos del hematocrito o volumen celular aglomerado (PCV), en comparación con bovinos parasitológicamente sanos (Cuglovici et al. 2010). Se ha reportado en Punjab (India), que valores de hemoglobina y de glucosa en sangre, se redujeron en bovinos infectados por $B$. bovis, en comparación con bovinos no infectados (Zulfiqar et al. 2012).

Tabla 3. Valores del cuadro hemático entre bovinos con y sin presencia de hematozoarios, en el Caribe colombiano.

\begin{tabular}{|c|c|c|c|c|c|}
\hline Variable & n & Media & D. estándar & V. mínimo & V. máximo \\
\hline \multicolumn{7}{|c|}{ Bovinos sin hematozoarios } \\
\hline Hematocrito & 185 & 34,17 & 6,46 & 19,00 & 53,00 \\
\hline Hemoglobina & 185 & 11,34 & 2,16 & 6,30 & 17,600 \\
\hline Eritrocitos & 185 & 56556 & 1085 & 310 & 8800 \\
\hline Leucocitos & 185 & 72572 & 2769 & 200 & 17900 \\
\hline \multicolumn{7}{|c|}{ Bovinos infectados con hematozoarios } \\
\hline Hematocrito & 125 & 31,50 & 6,26 & 14,00 & 45,00 \\
\hline Hemoglobina & 125 & 10,64 & 2,85 & 4,60 & 32,3 \\
\hline Eritrocitos & 125 & 5294400 & 1407486 & 2300 & 16100 \\
\hline Leucocitos & 125 & 8720 & 3,193 & 2500 & 17900 \\
\hline
\end{tabular}

Al comparar la CC entre bovinos con y sin hematozoarios (Tabla 4), el mayor número de bovinos infectados y no infectados, se concentró dentro de la categoría regular y mala, siendo las mayores frecuencias para los bovinos sanos, en comparación con los infectados; es posible que la CC este afectada por otras variables no evaluadas dentro de este estudio, como la disponibilidad y la calidad nutricional y el estado fisiológico. Se ha deducido que la presencia de hematozoarios no es evidencia concluyente para la CC, ya que depende de los factores anteriormente mencionados (Florio et al. 2012). En México, se dedujo que los hemoparásitos pueden representar problemas de salud y de producción animal (Rodríguez et al. 2000) y, en Vietnam, se concluyó que los hemoparásitos continúan siendo una amenaza para la industria ganadera (Sivakumar et al. 2013).
Este estudio, determinó la presencia clínica de anaplasmosis, babesiosis y, en menor frecuencia, tripanosomiasis, lo cual, puede repercutir en algunos parámetros de interés zootécnicos, como ganancia de peso. Igualmente, se hace necesario evaluar las medidas integrales de prevención y de control de vectores, como también caracterizar los costos y sus implicaciones sobre la producción animal.

Agradecimientos: Los autores agradecen a todos los ganaderos y asistentes técnicos, quienes permitieron la toma de las muestras en sus empresas ganaderas. Conflicto de intereses: Este manuscrito fue preparado y revisado con la participación de todos los autores, quienes declaramos que no existe conflicto de intereses que pongan en riesgo la validez de los resultados presentados. 
Tabla 4. Evaluación de la condición corporal en bovinos con y sin hematozoarios, en el Caribe colombiano.

\begin{tabular}{|l|c|c|c|c|c|c|}
\hline \multirow{2}{*}{$\begin{array}{l}\text { Condición } \\
\text { Corporal }\end{array}$} & \multicolumn{2}{|c|}{$\begin{array}{c}\text { Bovinos sin } \\
\text { hematozoarios }\end{array}$} & \multicolumn{2}{c|}{$\begin{array}{c}\text { Bovinos con } \\
\text { hematozoarios }\end{array}$} & \multicolumn{2}{c|}{ TOTAL } \\
\cline { 2 - 7 } & $\mathbf{n}$ & $\mathbf{\%}$ & $\mathbf{n}$ & $\mathbf{\%}$ & $\mathbf{n}$ & \% \\
\hline Excelente & 2 & 0,65 & 0 & 0 & 2 & 0,65 \\
\hline Buena & 3 & 0,97 & 4 & 1,29 & 7 & 2,26 \\
\hline Regular & 31 & 10,0 & 23 & 7,42 & 54 & 17,42 \\
\hline Mala & 153 & 48,06 & 98 & 31,61 & 247 & 79,67 \\
\hline TOTAL & 185 & 59,68 & 125 & 40,32 & 310 & 100 \\
\hline
\end{tabular}

\section{BIBLIOGRAFÍA}

1. AGUDO, L.; TAMASAUKAS, R.; SILVA, A.; SÁNCHEZ, J.; RON, J.; FERNÁNDEZ, M.; FLORIO, J.; VINTIMILLA, M.; COLMENARES, O.; RIVERA, S. 2009. Identificación y caracterización fenotípica de tipo bovino doble propósito trypanotolerante. Rev. Electrónica Veterinaria REDVET (España). 10(10). Disponible desde Internet en: http://www.veterinaria.org/revistas/ redvet/n101009/100910.pdf (con acceso 18/05/15).

2. AÑEZ, R.N.; ROMERO, O.; VALBUENA, H.; CRISANTE, G.; ROJAS, A.; BOLÍVAR, A.M.; AÑEZ, N. 2010. Detección de transmisión transplacentaria de Anaplasma marginale en bovinos asintomáticos. Rev. Cient. FCV-LUZ (Venezuela). 20(4):377-382.

3. BENAVIDES, O.E.; POLANCO, P.N.; VIZCAÍNO, O.; BETANCUR, H.O. 2012. Criterios y protocolos para el diagnóstico de hemoparásitos en bovinos. Rev. Cienc. Anim. (Colombia). 5:31-49.

4. BETANCOURT, J.A. 2011. Parasitología veterinaria y cambio climático. Biomédica (Colombia). 31(3):279282.

5. BIANCHIN, I.; CATTO, J.B.; KICHEL, A.N.; TORRES, R.A.A.; HONER, M.R. 2007. The effect of the control of endo- and ectoparasites on weight gains in crossbred cattle (Bos taurus taurus $\times$ Bos taurus indicus) in the central region of Brazil. Trop. Anim. Health Prod. 39:287-296.

6. BIRYOMUMAISHO, S.; RWAKISHAYA, E.K.; MELVILLE, S.E.; CAILLEAU, A.; LUBEGA, G.W. 2013. Livestock trypanosomosis in Uganda: parasite heterogeneity and anaemia status of naturally infected cattle, goats and pigs. Parasitol. Res. (Alemania). 112(4):14431450.
7. BOCK, R.; JACKSON, L.; De Vos, A.; JORGENSEN, W. 2004. Babesiosis of cattle. Parasitology. 129:S247269.

8. BOLÍVAR, A.M. 2013. Metodología diagnóstica para hemoparásitos dentro de la ganadería bovina con énfasis en la reacción en cadena de la polimerasa y su variante múltiple. Rev. Salud Anim. (Cuba). 35(1):1-9.

9. CADIOLI, F.A.; BARNABÉ P.A.; MACHADO, R.Z.; TEIXEIRA, M.C.; ANDRÉ, M.R.; SAMPAIO, P.H.; FIDÉLIS, J.O.L.; TEIXEIRA, M.M.; MARQUES, L.C. 2012. First report of Trypanosoma vivax outbreak in dairy cattle in São Paulo state, Brazil. Rev. Bras. Parasitol. Vet. (Brasil) 21(2):118-124.

10. CANEVER, M.F.; VIEIRA, L.L.; RECK, C.; RICHTER, L.; MILETTI, L.C. 2014. First evaluation of an outbreak of bovine babesiosis and anaplasmosis in Southern Brazil using multiplex PCR. Korean J. Parasitol. 52(5):507-511.

11. CORTÉS, J.A. 2010. Cambios en la distribución y abundancia de las garrapatas y su relación con el calentamiento global. Rev. Med. Vet. Zoot. 57:65-75.

12. CUGLOVICI, D.A.; BARTHOLOMEU, D.C.; REIS, C.J.L.; CARVALHO A.U.; RIBEIRO, M.F. 2010. Epidemiologic aspects of an outbreak of Trypanosoma vivax in a dairy cattle herd in Minas Gerais state, Brazil. Vet. Parasitol. (Brasil). 169(3-4):320-326.

13. FLORIO, L.J.; TAMASAUKAS, R.; RIVERA, S. 2012. Diagnóstico participativo de hemotrópicos en bovinos a nivel de pequeños productores y productoras de ganadería doble propósito en el sur del estado Aragua en la República Bolivariana de Venezuela. AICA (España). 2:163-170. 
14. FRISCH, J.E.; O‘NEILL, C.J; KELLY, M.J. 2000. Using genetics to control cattle parasites: the Rockhampton experience. Ind. J. Parasitol. 30:253-264.

15. GACHOHI, J.; BETT, B.; MURILLA, G. 2009. Factors influencing the prevalence of trypanosomosis in Orma Boran (trypanotolerant) and Teso zebu (trypanosusceptible) cattle crosses in Teso District, western Kenya. Livestock Res. Rural Development (Colombia). 21(12). Disponible desde Internet en: http:// www.Irrd.org/lrrd21/12/gach21216.htm (con acceso 18/05/15).

16. GILES, J.R.; PETERSON, A.T.; BUSCH, J.D.; OLAFSON, P.U.; SCOLES, G.A.; DAVEY, R.B.; POUND, J.M.; KAMMLAH, D.M.; LOHMEYER, K.H.; WAGNER, D.M. 2014. Invasive potential of cattle fever ticks in the southern United States. Parasites \& Vectors (Inglaterra). 7:189.

17. GONZÁLEZ, J.R.; MELÉNDEZ, R.D. 2007. Seroprevalencia de la tripanosomiasis y anaplasmosis bovina en el municipio Juan José Mora del Estado Carabobo, Venezuela, mediante la técnica de Elisa. Rev. Cient. (Venezuela). 17(5):7-10.

18. HERRERA, M.; SOTO, A.; URREGO, V.; RIVERA, G.; ZAPATA, M.; RÍOS, L. 2008. Frecuencia de hemoparásitos en bovinos del Bajo Cauca y Alto San Jorge, 2000-2005. Rev. MVZ Córdoba (Colombia). 13(3):1486-1494.

19. HOWARTH, J.A.; ROBY, T.O. 1972. Transmission of anaplasmosis by field collections of Dermacentor occidentalis Marx (Acarina: Ixodidae). Proc. US Animal Health Assoc. 76:98-102.

20. LATIMER, K.S.; MAHAFFEY, E.A.; PRASS, K.W. 2005. Duncan \& Prasse's Patología Clínica veterinaria 4 Edición. Multimedica Ed (Barcelona, España). 557p.

21. MÁRQUEZ, L.D. 2008. Residuos químicos en alimentos de origen animal: problemas y desafíos para la inocuidad alimentaria en Colombia. Rev. Corpoica Ciencia y Tecn. Agropec. (Colombia). 9(1):124-135.

22. MANTEROLA, C.; OTZEN, T. 2014. Estudios observacionales. Los diseños utilizados con mayor frecuencia en investigación clínica. Int. J. Morphol. 32(2):634-645.

23. OFICINA INTERNACIONAL DE SANIDAD ANIMAL OIE-. 2004. Anaplasmosis bovina. Manual de la OIE sobre enfermedades terrestres. 534-547. Disponi- ble desde Internet en: http:/www.oie.int/fileadmin/ Home/esp/Health_standards/tahm/2.04.01_Anaplasmosis_bovina.pdf (con acceso 18/05/15).

24. OLIVEIRA, M.C.S.; ALENCAR, M.M.; CHAGAS, A.C.S.; GIGLIOTI, R.; OLIVEIRA, H.N. 2009. Gastrointestinal nematode infection in beef cattle of different genetic groups in Brazil. Vet. Parasitol. 166:249-254.

25. OLIVEIRA, J.B.; MONTOYA, J.; ROMERO, J.J.; URBINA, A.; SOTO, B.N.; MELO, E.S.; RAMOS, C.A.; ARAÚJO, F.R. 2011. Epidemiology of bovine anaplasmosis in dairy herds from Costa Rica. Vet. Parasitol (Holanda). 177:359-365.

26. PRADA, S.G.; CRESPO, J. 2006. Determinación taxonómica de hemoparásitos y su prevalencia en búfalos de agua (Bubalus bubalis) en el Magdalena Medio, Colombia. Rev. Investigación (Colombia). 6(1):67-73.

27. QUIJADA, T.; CONTRERAS, J.; FORIANO, M. 1998. Seropositividad a Babesia bigemina en bovinos mestizos de Las Yaguas, Carora, Estado Lara, Venezuela. Veterinaria Tropical. 23(1):13-24.

28. REGASSA, A.; PENZHORN, B.; BRYSON, N. 2003. Attainment of endemic stability to Babesia bigemina in cattle on a South African ranch where non-intensive tick control was applied. Vet. Parasitol (Holanda). 116:267-274.

29. RÍOS, O.L.A.; ZAPATA, S.R.; REYES, J.; MEJÍA, J.; BAENA, A. 2010. Estabilidad enzoótica de babesiosis bovina en la región de Puerto Berrío, Colombia. Rev. Cient. FCV-LUZ (Venezuela). 20(5):485-492.

30. RISTIC, M.; McINTYRE, I. 1981. Diseases of Cattle in the tropics, Economic and zoonotic relevance. Marinus Nijhoff Publishers. 662p.

31. RODRÍGUEZ, V.R.I.; COB, G.L.A.; DOMÍNGUEZ, A.J.L. 2000. Hemoparásitos en bovinos, caninos y equinos diagnosticados en el Laboratorio de Parasitología de la Facultad de Medicina Veterinaria y Zootecnia de la Universidad Autónoma de Yucatán (1984-1999). Rev. Biomed. (México). 11:277-282.

32. ROSSI, S.M.S.; SIGALES, L.; ZAPATA, D. 2008. Inmunoensayo de capa fina (ICF) en el serodiagnóstico de la tripanosomiasis bovina causada por el Trypanosoma vivax. Rev. Fac. Cs. Vets. (Venezuela). 49(2):8189. 
33. SILVA, A.M.; ALENCAR, M.M.; REGITANO, L.C.A.; OLIVEIRA, M.C.S; BARIONI, JW. 2007. Artificial infestation of Boophilus microplus in beef cattle heifers of four genetic groups. Gen. Mol. Biol.30:1150-1155.

34. SIVAKUMAR, T.; LAN, D.T.; LONG, P.T.; YOSHINARI, T.; TATTIYAPONG, M.; GUSWANTO, A.; OKUBO, K.; IGARASHI I., INOUE, N.; XUAN, X.; YOKOYAMA, N. 2013. PCR detection and genetic diversity of bovine hemoprotozoan parasites in Vietnam. J. Vet. Med. Sci. (Japón). 75(11):1455-1462.

35. SOUZA, F.A.L.; BRAGA, J.F.V.; PIRES.; LIDIANY, V.L.; CARVALHO, C.J.S.; COSTA, E.A.; MÚCIO, F.B.; RIBEIRO, M.F.V.; SANTOS, R.L.; SILVA, M.M.S. 2013. Babesiosis and anaplasmosis in dairy cattle in Northeastern Brazil. Pesq. Vet. Bras. 33(9):10571061.

36. SUÁREZ, G.; GARCÍA, F.; ROMÁN, D.; CORONADO, A.; PERRONE, T.; REYNA, A.; PARRA, N. 2009. Factores de riesgo asociado a la tripanosomosis bovina en explotaciones ganaderas de Venezuela. Zootecnia Trop. 27(4):363-372.

37. THORNTON, P.K.; VAN DE STEEG, J.; NOTENBAERT, A.; HERRERO, M. 2009. The impacts of climate change on livestock and livestock systems in devel- oping countries: A review of what we know and what we need to know. Agric. Syst. 101:113-127.

38. VIZCAÍNO, O. 2011. Anaplasma marginale: noxa de rickettsias, antigénica, inmunológica y económicamente importante en bovinos de áreas intertropicales. Biomédica (Colombia). 31:18-31.

39. WICKWITE, K.B.; KOCAN, K.M.; BARRON, S.J.; SMITH, R.D.; HAIR, J.A. 1987. Infectivity of three Anaplasma marginale isolates for Dermacentor andersoni. Am. J. Vet. Res 48(1):96-99.

40. ZINTL, A.; MULCAHY, G.; SKERRETT, H.E.; TAYLOR, S.M.; GRAY, J.S. 2003. Babesia divergens, a bovine blood parasite of veterinary and zoonotic importance. Clinical Microbiol. Reviews. 16(4):622-636.

41. ZULFIQAR, S.; SHAHNAWAZ, S.; ALI, M.; BHUTTA, A.M.; IQBAL, S.; HAYAT, S.; QADIR, S.; LATIF, M.; KIRAN, N.; SAEED, A.; ALI, M.; IQBAL, F. 2012. Detection of Babesia bovis in blood samples and its effect on the hematological and serum biochemical profile in large ruminants from Southern Punjab. Asian. Pac. J. Trop. Biomed. (China). 2(2):104-108.

Recibido: Abril 10 de 2015

Aceptado: Noviembre 3 de 2015

\section{Cómo Citar}

Calderón, A.; Martínez, N.; Iguarán, H. 2016. Frecuencia de hematozoarios en bovinos de una región del caribe colombiano. Rev. U.D.C.A Act. \& Div. Cient. 19(1): 131-138. 